\title{
Health Systems Strengthening Interventions for Perinatal Common Mental Disorders and Experiences of Domestic Violence in Cape Town, South Africa: Protocol for a Pilot Implementation Study
}

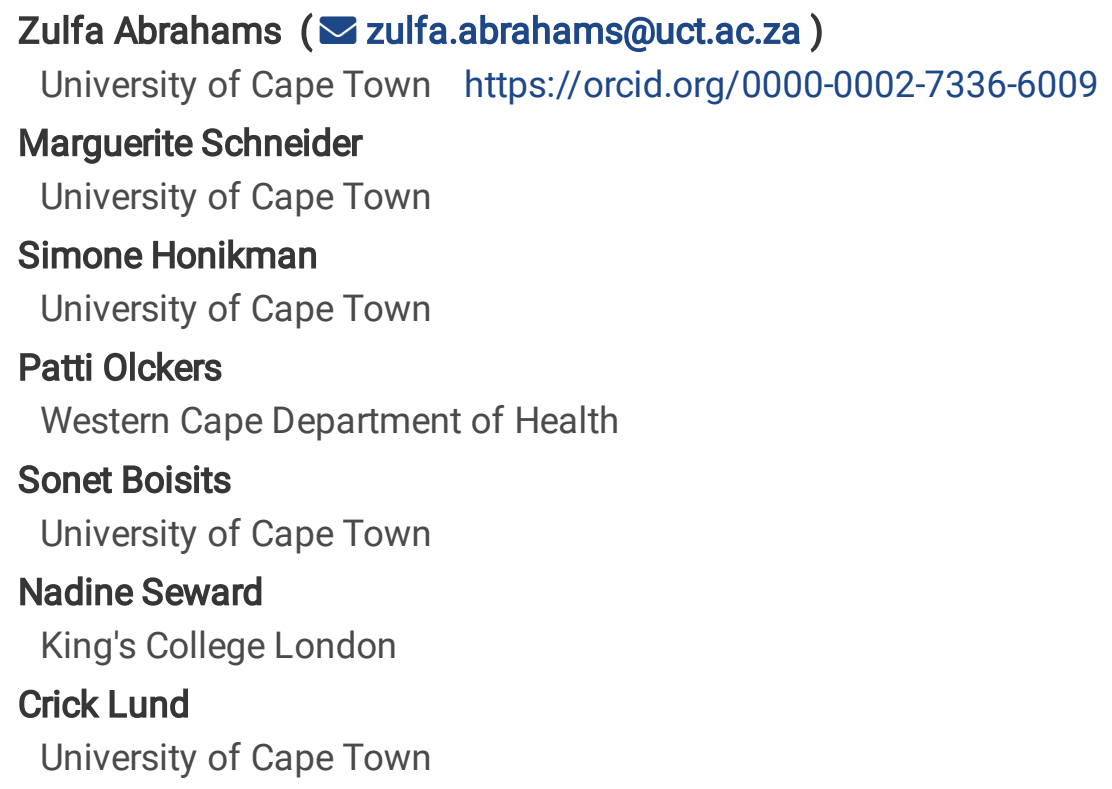

Keywords: common mental disorders, domestic violence, detection, counselling, implementation science, health system strengthening

Posted Date: October 18th, 2021

DOI: https://doi.org/10.21203/rs.3.rs-956295/v1

License: (c) (i) This work is licensed under a Creative Commons Attribution 4.0 International License. Read Full License 


\section{Abstract}

Background: During the perinatal period, common mental disorders (CMDs) such as depression and anxiety are highly prevalent, especially in low resource settings, and are associated with domestic violence, poverty and food insecurity. Perinatal CMDs have been associated with several adverse maternal and child outcomes. While the Department of Health in South Africa provides healthcare workers with the tools to detect psychological distress and experiences of domestic violence, few healthcare workers routinely screen pregnant women at clinic visits, citing discomfort with mental health issues and the lack of standardised referral pathways as the key barriers. The aim of this study is to select and evaluate a set of health systems strengthening (HSS) interventions aimed at improving the care and outcomes for perinatal women with CMDs and experiences of domestic violence, attending public healthcare facilities in Cape Town.

Methods: This study consists of a pre-implementation, development, and implementation phase. Contextual barriers identified during the pre-implementation phase included poor patient knowledge and health-seeking behaviour, high levels of stigma, and poor detection, referral and treatment rates. Implementation science determinant frameworks were applied to findings from the pre-implementation phase to identify determinants and gaps in delivering high-quality evidence-informed care. A participatory Theory of Change workshop was used to design a HSS programme, consisting of awareness raising, detection, referral, and treatment. HSS interventions selected to support the delivery of the HSS programme includes training, health promotion, change to the healthcare environment, task-sharing, audit and feedback and performance monitoring. The implementation phase will be used to assess several implementation and clinical outcomes associated with delivery of the HSS programme, which will be piloted at three healthcare facilities. Qualitative and quantitative methods will be used to evaluate the implementation and clinical outcomes.

Discussion: This pilot implementation study will inform us about a range of implementation and clinical outcome measures that are relevant for assessing health systems strengthening interventions for perinatal women with depression, anxiety or experiences of domestic violence in low resource settings. Lessons learnt from the pilot study will be incorporated into the design of a cluster randomised control trial for which further funding will be sought.

\section{Background}

Common mental disorders (CMD) such as depression and anxiety, are highly prevalent during the perinatal period (pregnancy and the first year postpartum) in the Western Cape, South Africa. A facility-based study in Hanover Park, a low-income, residential suburb in Cape Town [1], reported that $21 \%$ of pregnant women were diagnosed with depression and $23 \%$ were diagnosed with anxiety [2]. Similar results were obtained from a study in Khayelitsha - a large peri-urban township settlement in Cape Town - where 19\% of pregnant women screened positive for a CMD [3].

In low-resource settings such as these, the prevalence of domestic violence, poverty and food insecurity is high. Interpersonal violence is ranked second in its contribution to the burden of disease in South Africa, and among women, intimate partner violence accounts for $62 \%$ of the burden of interpersonal violence [4]. Recent studies among perinatal women in Cape Town reported that approximately $15 \%$ of pregnant women experienced domestic violence during the past 12 months, more than $40 \%$ lived in food insecure households, and that food insecurity and domestic violence increased the risk of subsequently developing a CMD [2,5-7]. Furthermore, perinatal CMDs have been associated with several adverse consequences for both mother and child, including preterm birth, low birthweight and diminished mother-infant bonding $[8,9]$.

Following South Africa's adoption of the Mental Health Policy Framework and Strategic Plan 2013-2020 [10], which states that mental health should be integrated into maternal and child health platforms, maternal and child health were 
identified by the National Department of Health $(\mathrm{DoH})$ as key priority areas. With this in mind, an updated version of the Maternity Case Record (MCR) - a record book given to pregnant women and used to record all clinical details of the pregnancy [11] - containing a three-item mental health screening questionnaire [12], was released. The mental health screening questionnaire, which measures psychological distress experienced during the prior two weeks, was also incorporated into the Practical Approach to Care Kit (PACK) - Primary Care Guide for the Adult [13] - a clinical decision support tool for primary care workers. PACK includes guidelines for the assessment of traumatised or abused patients, and the MCR allows for healthcare workers to record the presence of abuse. However, even when provided with tools to detect psychological distress and experiences of domestic violence, few healthcare workers routinely screen pregnant women at clinic visits, citing discomfort with mental health issues and the lack of standardised referral pathways as the key barriers [14].

To assist the $\mathrm{DoH}$, several models have been developed to provide screening and mental health care during the perinatal period in the Western Cape. The Perinatal Mental Health Project provides a successful screening and counselling service at one Midwife Obstetric Unit (MOU) in Cape Town [15]. The Africa Focus on Intervention Research for Mental Health (AFFIRM) randomized controlled trial developed and evaluated a structured manualised task sharing counselling intervention for antenatal depression at two MOUs in Khayelitsha, Cape Town in 2014-2016, delivered by Community Health Workers (CHWs) [16]. The trial showed that there was a small but significant effect of the counselling intervention on psychological distress at three months postnatal, compared to enhanced usual care (three supportive monthly phone calls) [3]. The effect became more significant at the 12 months postnatal assessment, suggesting that the counselling intervention has longer term benefits on managing depressive symptoms compared to the phone calls. Women in both intervention and control arms showed a substantial reduction in depression symptoms into the postnatal period. Following presentation of the preliminary AFFIRM trial results in January 2017, senior managers in the Western Cape DoH were supportive of scaling up a counselling service for antenatal depression in MOUs and Basic Antenatal Care (BANC) clinics in the Cape Town metropolitan area.

To that end, the Health Systems Strengthening in sub-Saharan Africa (ASSET) study sought to collaborate with the Western Cape DoH to select and evaluate a set of health systems strengthening (HSS) interventions aimed at improving the quality of care and clinical outcomes for perinatal women with CMDs and experiences of domestic violence, attending public healthcare facilities in Cape Town. To help better understand how this study can be adapted for scaleup, we aim to assess the influence of the HSS interventions on important clinical and implementation outcomes such as feasibility, acceptability, fidelity, and appropriateness throughout the implementation process.

\section{Methods}

This is a multi-phase study, consisting of a (1) pre-implementation phase, (2) development phase and (3) implementation phase. The first two phases of the study have already been completed, and the third phase is currently in progress with enrolment in the study not yet completed.

\section{Implementation science}

Implementation science determinant and evaluation frameworks were used throughout the study. At the end of the preimplementation phase of the study, we used determinant frameworks to identify the barriers and/or enablers that we may have missed that would further inform the selection of any additional HSS interventions [17]. Specifically, the Theoretical Domains Framework (TDF) [18] and the Context and Implementation of Complex Interventions Framework (CICl) [19] were used to identify determinants that could potentially influence implementation efforts throughout the implementation process. The Effective Practice and Organisation of Care (EPOC) taxonomy of implementation strategies was used to select relevant HSS interventions [20]. The conceptual framework for implementation outcomes 
by Proctor et al. [21] was used to select relevant implementation outcomes. The TIDieR checklist and 'Getting messier with TIDieR' template [22] were used to describe the HSS programme in its entirety.

\section{Pre-implementation phase}

The pre-implementation phase [23] took place in four MOUs and in the ten non-profit organisations (NPOs) mandated to provide facility- and community-based support to patients attending those facilities. The MOUs were purposively selected by DoH managers to represent each of the four sub-districts in the Cape Metropolitan health district in Cape Town. Supplementary file 1 describes the study designs, setting, data collection tools, and participants involved in the pre-implementation phase of the study. In each facility we undertook (i) a situation analysis, (ii) a cross-sectional survey among pregnant women attending the MOUs for their first antenatal visit, (iii) qualitative interviews with pregnant women experiencing psychological distress or experiences of domestic violence and (iv) qualitative interviews with healthcare workers. These methods and findings have been published elsewhere [14].

\section{Development phase}

The development phase involved (i) designing a HSS programme, (ii) selecting HSS interventions to support the delivery of the HSS programme and address the contextual barriers identified during the pre-implementation phase, (iii) developing the processes and tools to deliver the HSS programme and evaluate the HSS interventions, and (iv) piloting the HSS programme at one facility.

\section{Designing the HSS programme}

The design of the HSS programme was guided by a Theory of Change (ToC) workshop. ToC workshops are a participatory process whereby a group of stakeholders agree on the long-term outcome the programme would like to achieve and identify the short- and medium-term outcomes needed to achieve it. The outcomes are graphically presented in a causal framework together with the assumptions of what needs to take place to achieve the outcomes, the contextual factors which influence the outcomes and the indicators needed to measure the achievement of the outcomes [26].

We facilitated a ToC workshop with approximately $40 \mathrm{DoH}$ managers and clinical staff working in various programmes within the four sub-districts in the Cape Metropolitan health district. Following the presentation of the preimplementation phase findings, workshop participants discussed and agreed on the long-term outcome the programme aimed to achieve - improved coverage of maternal mental health services. Thereafter participants worked in small groups to clarify what existed and to identify the short- and medium-term outcomes, as well as the actions and assumptions needed. Small group discussions were fed back and discussed in the bigger group. Group discussions and feedback sessions were recorded and used to guide the development of the ToC Diagram (Figure 1). Several contextual barriers were identified including poor patient knowledge of mental health and domestic violence, high levels of stigma, poor mental health seeking behaviour, and poor detection, referral and treatment rates.

The ToC diagram guided the development of a process map (Figure 2) to visually describe the processes and actions needed by healthcare workers to achieve the long-term outcome. Over a three-month period, an iterative process of presenting the process map to various groups of sub-district, facility, and community-level healthcare workers and managers for feedback, adjustment and approval was followed.

The approved HSS programme consisted of four main components - awareness raising, detection, referral and treatment. To operationalise the HSS programme, the following activities would be needed (i) health promotion officers would deliver health promotion and awareness talks to pregnant women, (ii) antenatal care (ANC) nurses would detect 
and refer pregnant women with CMDs and experiences of domestic violence, and (iii) CHWs would deliver a structured counselling programme to pregnant women with mild to moderate symptoms of CMDs.

\section{Selecting the HSS interventions and implementation outcomes}

Findings from the pre-implementation phase of the study were reviewed at an annual ASSET meeting [17]. The TDF [18] and the $\mathrm{CICl}$ framework [19] were used to identify contextual and behavioural determinants that could influence the implementation of HSS interventions and implementation outcomes that may have been missed in the ToC workshop. If there were determinants not identified in the pre-implementation phase, these were accounted for at this stage. The HSS interventions that were selected in the ToC workshop were also labelled according to the EPOC taxonomy [18, 20].

Finally, we ensured that implementation outcomes were aligned with the selected HSS interventions. Table 1 provides on overview of the contextual barriers that were identified, the HSS interventions that were selected and the implementation outcomes that will be assessed. 
Table 1

Contextual barriers identified, HSS interventions selected and implementation outcomes to be assessed during the implementation phase

$\begin{array}{lll}\begin{array}{l}\text { Contextual HSS interventions } \\ \text { barriers }\end{array} & \text { Implementation outcomes }\end{array}$

Poor patient $\quad$ Training - of Health Promotion (HP) Officers to knowledge and health seeking behaviour; high levels of stigma
Low levels of detection
Health promotion - delivery of daily health promotion talks to pregnant women deliver health promotion talks
Acceptability, appropriateness, satisfaction and feasibility of talks - qualitative interviews with pregnant women and HP Officers

Adoption and fidelity of talks - observation of talks and completion of a checklist

Sustainability of daily talks - qualitative interviews with HP Officers

Effectiveness of talks - change in knowledge, attitudes and health seeking behaviour of pregnant women

Acceptability, appropriateness, satisfaction and feasibility of detection process qualitative interviews with pregnant women and ANC nurses

Adoption and fidelity of detection process review of patient files and documents used to record detection rates

Sustainability of the detection process qualitative interviews with ANC nurses

Audit and feedback - screening rates assessed and performance summarised and discussed at bi-monthly meetings

Poor linkage to care

Referral systems - development of standardised referral pathways

Training - of ANC nurses to refer pregnant women with symptoms of common mental disorders and experiences of abuse for treatment

Audit and feedback - referral rates assessed and performance summarised and discussed at bi-monthly meetings

Limited availability of treatment
Task-sharing - psychological counselling program to be delivered by lay healthcare workers

Training - of lay healthcare workers to deliver psychological counselling

Delivery of individual-level care - lay healthcare workers deliver psychological counselling

Change to healthcare environment psychological counselling delivered in patients' homes or at off-site venues

Audit and feedback - counselling rates assessed and performance summarised and discussed at bi-monthly meetings

Performance monitoring - supervisors monitor delivery of counselling
Acceptability, appropriateness, satisfaction and feasibility of referral process qualitative interviews with pregnant women and ANC nurses

Adoption and fidelity of referral process review of patient files and documents used for referral

Sustainability of the referral process qualitative interviews with ANC nurses

Acceptability, appropriateness, satisfaction and feasibility of counselling program qualitative interviews with pregnant women and lay healthcare workers

Fidelity of counselling delivery counselling competence of lay healthcare workers

Adoption, penetration and sustainability of counselling program - review documents used for referral; qualitative interviews with pregnant women and lay healthcare workers 
Developing a training model

To ensure sustainability of the HSS programme, a cascaded training model (Supplementary file 2) was developed, whereby the ASSET team provided master training workshops at centralised locations. Three master training workshops were held with selected DoH staff members: (i) facility-based psychiatric nurses received training on health promotion and awareness, (ii) facility PACK trainers received training on detection and referral, and (iii) community-based services (CBS) trainers from each of the sub-districts (whose primary role was to provide on-going training to community-based healthcare workers on a range of topics) and NPO managers from the supporting NPOs received the counselling and supervision training. Master trainers were tasked with providing training to small groups of facility- and communitybased healthcare workers whose role would include implementing the selected HSS interventions.

Addressing poor patient knowledge and health seeking behaviour

Training and health promotion were selected as the HSS interventions to address poor patient knowledge of mental health and domestic violence, poor health seeking behaviour, and the high levels of stigma among patients. The awareness raising component of the HSS programme will consist of health promotion officers or other lay healthcare workers providing daily, 5-7-minute talks to groups of pregnant women in waiting areas at facilities. Information to be covered during the talks will include the signs, symptoms, risk factors and consequences of depression, anxiety and experiences of domestic violence as well as the treatment options available.

Facility-based psychiatric nurses who were the recipients of the master training, will be tasked with providing cascaded training to health promotion officers or other lay healthcare workers at facilities. Master trainers will be provided with a Health Promotion and Awareness of Maternal Mental Health Training Manual [27] to guide the delivery of the training content. Healthcare workers who will be trained to implement the health promotion talks will be provided with an A3 size, colour flipchart to guide the delivery of the talk.

Addressing low levels of detection

Training, delivery of individual-level care and audit and feedback were selected as the HSS interventions to address the low levels of detection. ANC nurses will be trained to screen all pregnant women for symptoms of CMDs and experiences of domestic violence as part of routine care, using the mental health screening questionnaire [12] available in the MCR [11] and the PACK guidelines [28]. The training material was developed in conjunction with the Knowledge Translation Unit (KTU), a clinical research unit at the University of Cape Town, that led the development of PACK. The PACK guide was used as the foundation for the detection and referral process. A PACK Antenatal Women and Mental Health Module [29] was developed to strengthen the mental health component of routinely provided antenatal care. Four case studies formed the backbone of the module, complemented by HSS discussions, completion of relevant stationery and a focus on effective communication strategies. PACK facility trainers were the recipients of the master training and will be tasked with training ANC nurses at their facilities. The cascaded training will consist of four, weekly, 2-hour training sessions.

During the implementation phase, audit and feedback will be used to assess the screening rates at facilities and provide feedback to the relevant ANC nurses and their managers at bi-monthly meetings.

Addressing poor linkage to care

Referral systems, training and audit, and feedback were selected as the HSS interventions to address the poor linkage to care. Standardised referral pathways were developed. PACK Facility Trainers (facility-based healthcare workers who are 
PACK trained and assigned to deliver PACK training to healthcare workers in their own facility) were the recipients of the master training and will be tasked with training ANC nurses at their facilities. ANC nurses will be trained to assess the severity of symptoms in pregnant women who screen positive, and to use the standardised referral pathways to link them to care. Pregnant women with mild to moderate symptoms of depression will be referred to CHWs, while women with severe symptoms of depression will be referred to healthcare workers providing specialised care such as Medical Officers, Psychiatric Nurses, and Psychologists. Pregnant women experiencing domestic violence will be referred to a Social Worker for support.

During the implementation phase, audit and feedback will be used to assess the referral rates at facilities and provide feedback to the relevant ANC nurses and their managers at bi-monthly meetings.

Addressing the limited availability of treatment

Several HSS interventions were selected to address the limited availability of treatment, including task-sharing, training, delivery of individual-level care, change to the healthcare environment, audit and feedback, and performance monitoring. A task-sharing psychological counselling programme was developed, to be delivered by $\mathrm{CHWs}$ to pregnant women with mild to moderate symptoms of depression or anxiety. It consists of three, 45-minute, structured, individuallevel counselling sessions using problem-solving therapy, delivered weekly by CHWs, in patients' homes [30]. The development of the psychological counselling programme was informed by a manual review of counselling interventions, semi-structured interviews with healthcare workers and pregnant women, and finally through several stakeholder engagement meetings. CHWs will be supervised and supported by Outreach Team Leaders (OTLs) to reinforce the counselling skills, ensure fidelity to the psychological counselling and manage difficult cases.

CBS trainers employed in each sub-district were selected as the recipients of the master training. Master trainers received a 4-day training workshop, where they were provided with a Counselling Skills for Community Health Workers [31] training manual and a Maternal Mental Health Counselling Support Guide to assist them in delivering cascaded training on (i) counselling, and (ii) supervision and support. The cascaded counselling training will be delivered over three days to CHWs and OTLs and consist of five sections: (i) understanding depression, anxiety and experiences of domestic violence, (ii) basic counselling skills, (iii) patient assessment, (iv) 3-session counselling intervention, and (v) coping skills. The cascaded supervision and support training session will be delivered to OTLs during a one-day training which will include (i) counselling support styles and skills, and (ii) the use of individual and group support. CHWs and OTLs will be provided with a Reference Guide for Community Health Workers, in addition to the Counselling Skills for Community Health Workers training manual to support the delivery of the counselling intervention.

During the implementation phase, audit and feedback will be used to assess the counselling rates and provide feedback to the relevant $\mathrm{CHWs}$, OTLs and their managers at bi-monthly meetings. OTLs will also observe the delivery of the counselling sessions to monitor the performance of the CHWs.

\section{Pilot study}

The intervention processes and tools were piloted at one of the pre-implementation phase MOUs. Lessons learnt and feedback received from healthcare workers involved in the pilot study were incorporated into the final design and used to develop a referral pathway diagram (Figure 3).

\section{Implementation phase}

The implementation phase will be used to deliver the HSS interventions selected to improve awareness, detection, referral and treatment of CMDs and experiences of domestic violence in perinatal women attending public healthcare facilities. Both clinical outcomes and implementation outcomes will be assessed. 


\section{Setting and participants}

Three healthcare facilities will be purposively selected. Study sites will include a combination of (i) MOUs and BANC clinics situated in low-resource settings, (ii) provide care to women of mixed ancestry and Black African women, (iii) represent three of the four sub-districts within the Cape Metropolitan health district, and (iv) provide care to $\geq 60$ pregnant women attending the facility for their first antenatal clinic appointment each month. All NPOs providing community-based support to the selected facilities will automatically be included.

The participants will include perinatal women attending the healthcare facilities, and sub-district, facility and community-based healthcare workers. The following cadres of healthcare workers will be included: CBS trainers from each sub-district; PACK facility trainers based at each facility; psychiatric nurses, ANC nurses, and health promotion officers based at each facility; CHWs and OTLs employed by each NPOs supporting the selected facilities.

\section{Study designs}

The following study designs will be used to assess the implementation and clinical outcomes of the intervention: (i) healthcare worker survey, (ii) patient survey, (iii) observation of health promotion talks, (iv) cohort study, (v) patient file reviews, (vi) documentation review, (viii) counselling competence, and (vii) qualitative assessments. Table 2 provides an overview of the study designs and objectives, data collection instruments, participants to be recruited and the timing of data collection. 
Table 2

Implementation phase study designs, study objectives, data collection tools, study participants and timing of data collection

\begin{tabular}{|c|c|c|c|c|}
\hline Study design & Study objectives & Data collection tools & Participants & $\begin{array}{l}\text { Timing of data } \\
\text { collection }\end{array}$ \\
\hline $\begin{array}{l}\text { Healthcare } \\
\text { worker survey }\end{array}$ & $\begin{array}{l}\text { Assess changes in } \\
\text { healthcare workers } \\
\text { knowledge of } \\
\text { mental illness and } \\
\text { domestic violence } \\
\text { and their attitudes } \\
\text { towards people } \\
\text { with mental health } \\
\text { disorders and } \\
\text { experiences of } \\
\text { domestic violence }\end{array}$ & $\begin{array}{l}\text { Self-administered questionnaires: } \\
\text { Organisational readiness for } \\
\text { implementing change [32]; } \\
\text { Bespoke knowledge } \\
\text { questionnaire; Mental IIIness: } \\
\text { Clinicians' Attitudes Scale [33]; } \\
\text { Professional Quality of Life Scale } \\
\text { [34]; Effort-Reward Imbalance } \\
\text { scale [35] }\end{array}$ & $\begin{array}{l}\text { All healthcare } \\
\text { workers involved } \\
\text { in the } \\
\text { intervention - } \\
\text { CBS trainers, } \\
\text { NPO managers, } \\
\text { PACK facility } \\
\text { trainers, } \\
\text { psychiatric } \\
\text { nurses, ANC } \\
\text { nurses, OTLs, } \\
\text { CHWs, health } \\
\text { promotion } \\
\text { officers, HIV } \\
\text { counsellors, } \\
\text { nursing } \\
\text { assistants }\end{array}$ & $\begin{array}{l}\text { Two time- } \\
\text { points -before } \\
\text { receiving } \\
\text { training, and at } \\
\text { the end of the } \\
\text { implementation } \\
\text { phase }\end{array}$ \\
\hline Patient survey & $\begin{array}{l}\text { Assess changes in } \\
\text { pregnant women's } \\
\text { knowledge of } \\
\text { mental illness and } \\
\text { domestic violence } \\
\text { and their attitudes } \\
\text { towards people } \\
\text { with mental health } \\
\text { disorders and } \\
\text { experiences of } \\
\text { domestic violence }\end{array}$ & $\begin{array}{l}\text { Self-administered bespoke } \\
\text { questionnaire consisting of } 16 \\
\text { questions }\end{array}$ & $\begin{array}{l}\text { Pregnant women } \\
\text { attending MOUs } \\
\text { and BANC clinics }\end{array}$ & $\begin{array}{l}\text { Two time- } \\
\text { points - before } \\
\text { the delivery of } \\
\text { health } \\
\text { promotion } \\
\text { talks, after } \\
\text { delivery of the } \\
\text { health } \\
\text { promotion talks }\end{array}$ \\
\hline $\begin{array}{l}\text { Observation of } \\
\text { health } \\
\text { promotion } \\
\text { talks }\end{array}$ & $\begin{array}{l}\text { Assess uptake, } \\
\text { fidelity, penetration } \\
\text { and sustainability } \\
\text { of health } \\
\text { promotion talks }\end{array}$ & $\begin{array}{l}\text { Checklist to be completed by } \\
\text { fieldworkers observing the talks }\end{array}$ & & $\begin{array}{l}\text { During the } \\
\text { delivery of } \\
\text { health } \\
\text { promotion talks }\end{array}$ \\
\hline Cohort study & $\begin{array}{l}\text { Assess the } \\
\text { presence of, and } \\
\text { risk factors for } \\
\text { CMDs and } \\
\text { experiences of } \\
\text { domestic violence; } \\
\text { assess whether the } \\
\text { HSS interventions } \\
\text { to improve } \\
\text { awareness, } \\
\text { detection, referral } \\
\text { and treatment } \\
\text { resulted in } \\
\text { improvement in } \\
\text { clinical outcomes }\end{array}$ & $\begin{array}{l}\text { Interviewer administered } \\
\text { questionnaires: Edinburgh } \\
\text { postnatal depression scale [25]; } \\
\text { Psychological distress screening } \\
\text { tool [12]; Bespoke questionnaire to } \\
\text { assess domestic violence; } \\
\text { Household food insecurity and } \\
\text { access scale [36]; Composite } \\
\text { abuse scale [37]; WHO disability } \\
\text { assessment schedule [38]; } \\
\text { Multidimensional scale of } \\
\text { perceived social support [39]; } \\
\text { Mother-to-Infant bonding scale } \\
\text { [40] }\end{array}$ & $\begin{array}{l}\text { Pregnant and } \\
\text { postnatal women } \\
\text { attending } \\
\text { antenatal care } \\
\text { clinics }\end{array}$ & $\begin{array}{l}\text { Three time- } \\
\text { points - (1) } \\
\text { when pregnant } \\
\text { women are } \\
\text { recruited, (2) } \\
\text { when } \\
\text { participants are } \\
36 \text { weeks } \\
\text { pregnant, and } \\
\text { (3) six weeks } \\
\text { after } \\
\text { participants } \\
\text { have given } \\
\text { birth }\end{array}$ \\
\hline $\begin{array}{l}\text { Patient file } \\
\text { reviews }\end{array}$ & $\begin{array}{l}\text { Assess changes in } \\
\text { detection and } \\
\text { referral rates of } \\
\text { pregnant women } \\
\text { with CMDs and } \\
\text { experiences of } \\
\text { domestic violence }\end{array}$ & Maternity case record [11] & $\begin{array}{l}\text { Pregnant and } \\
\text { postnatal women } \\
\text { attending MOUs } \\
\text { and BANC clinics }\end{array}$ & $\begin{array}{l}\text { Weekly during } \\
\text { the } \\
\text { implementation } \\
\text { phase }\end{array}$ \\
\hline
\end{tabular}




\begin{tabular}{|c|c|c|c|c|}
\hline Study design & Study objectives & Data collection tools & Participants & $\begin{array}{l}\text { Timing of data } \\
\text { collection }\end{array}$ \\
\hline $\begin{array}{l}\text { Documentation } \\
\text { review }\end{array}$ & $\begin{array}{l}\text { Assess coverage of } \\
\text { the detection, } \\
\text { referral and } \\
\text { treatment } \\
\text { interventions }\end{array}$ & $\begin{array}{l}\text { Bespoke patient registers and } \\
\text { tracking forms }\end{array}$ & $\begin{array}{l}\text { ANC nurses and } \\
\text { OTLs }\end{array}$ & $\begin{array}{l}\text { Daily during the } \\
\text { intervention } \\
\text { period }\end{array}$ \\
\hline $\begin{array}{l}\text { Counselling } \\
\text { competence }\end{array}$ & $\begin{array}{l}\text { Assess fidelity to } \\
\text { the structured } \\
\text { counselling format } \\
\text { and content }\end{array}$ & $\begin{array}{l}\text { Enhancing assessment of } \\
\text { common therapeutic factors tool } \\
\text { [41]; Bespoke counselling } \\
\text { evaluation form }\end{array}$ & $\begin{array}{l}\text { Community } \\
\text { health workers } \\
\text { (CHWs) }\end{array}$ & $\begin{array}{l}\text { During the last } \\
2 \text { months of } \\
\text { the intervention }\end{array}$ \\
\hline $\begin{array}{l}\text { Qualitative } \\
\text { assessments }\end{array}$ & $\begin{array}{l}\text { Assess the } \\
\text { acceptability, } \\
\text { appropriateness, } \\
\text { satisfaction and } \\
\text { feasibility of the } \\
\text { selected HSS } \\
\text { interventions }\end{array}$ & $\begin{array}{l}\text { Topic guides for key informant } \\
\text { interviews and focus group } \\
\text { discussions }\end{array}$ & $\begin{array}{l}\text { Pregnant and } \\
\text { postnatal women } \\
\text { involved in the } \\
\text { intervention } \\
\text { Healthcare } \\
\text { workers involved } \\
\text { in the } \\
\text { intervention }\end{array}$ & $\begin{array}{l}\text { During the last } \\
3 \text { months of } \\
\text { the intervention }\end{array}$ \\
\hline
\end{tabular}

Healthcare worker survey

All healthcare workers involved in the implementation of a HSS intervention will be asked to complete a selfadministered survey questionnaire prior to receiving training. The questionnaire will be available in participants' home language and take 30 to 45 minutes to complete. In the last month of the study, healthcare workers who were actively involved in the intervention will be asked to complete the same survey questionnaire. We expect the number of healthcare workers linked to each facility to differ but estimate that between 10 and 20 healthcare workers are linked to each of the MOUs and BANC clinics. We intend to approach all healthcare workers and anticipate that at least $70 \%$ (i.e., 7-10 healthcare workers per facility) will agree to participate in the survey. The survey will be used to evaluate whether delivering the various HSS interventions resulted in a change in healthcare workers mental health knowledge, attitudes towards persons with mental illness, their psycho-social well-being and their quality of life.

\section{Patient survey}

During the baseline data collection period (prior to delivering the HSS interventions), pregnant women awaiting their routine tests and consultations will be approached and asked to complete a self-administered survey questionnaire, available in their home language. During the implementation phase, pregnant women who received the health promotion talk will be asked to complete the same survey questionnaire immediately after the talk has been delivered. The survey questionnaire will consist of a few demographic questions; questions about their knowledge of depression, anxiety and domestic violence; questions on attitudes towards people with mental illness and experiences of domestic violence; and questions on their health seeking behaviour. We anticipate that 15 to 30 pregnant women will be willing to complete the survey after a talk, and plan on administering the survey daily during the two weeks of baseline data collection, and at least once per week during the implementation phase. The survey will be used to assess the effect of the health promotion talks in changing the mental health knowledge, attitudes and health seeking behaviour of perinatal women.

Observation of health promotion talks 
Fieldworkers based at facilities will be tasked with observing the delivery of the health promotion talks and completing a checklist to assess (i) the extent to which the talks are adopted at facilities, (ii) whether the talks are being delivered as intended, and (iii) the extent to which the talks are accessed by the pregnant women.

\section{Cohort study}

At baseline, pregnant women attending the clinic for their first antenatal visit will be screened by fieldworkers, in their home language, for the presence of CMDs and experiences of domestic violence. All women who screen positive - i.e. score $\geq 13$ on the EPDS, or $\geq 2$ on the bespoke violence screening tool - will be invited to participate in the cohort study. A random sample of $33 \%$ of pregnant women who screen negative will also be invited to participate in the cohort study. Fieldworkers will administer several questionnaires assessing risk factors for CMDs and the frequency of domestic violence. The presence of, and risk factors for CMDs and experiences of domestic violence in cohort participants will be assessed again at the 36-week gestation and the 6-week postnatal follow-up time-points. Changes in clinical outcomes and their risk factors will be assessed by comparing the 36-week gestation and the 6-week postnatal outcomes to the baseline outcomes.

The cohort study will be used to (i) assess the presence of, and risk factors for CMDs and experiences of domestic violence in pregnant women, and (ii) to assess whether the HSS interventions resulted in improvement in clinical outcomes. It will be based on the proportion of women showing a clinically significant improvement as measured by the EPDS. Women with an EPDS score of $\geq 13$ at baseline will be classified as having symptoms of CMD. Clinically significant improvement will be classified as having a 50\% reduction in EPDS scores measured at the 36-week gestation and 6-week postnatal follow-up time-points, compared to baseline. The study will also assess the feasibility of administering these instruments and effectively following up perinatal women in these communities, for a potential future $\mathrm{CRCT}$. The study will also assess the feasibility of administering these instruments and effectively following up perinatal women in these communities, for a potential future cRCT.

Sample size calculations were used to determine the number of participants needed for a cluster randomised control trial $(\mathrm{CRCT})$. Based on a level of significance $($ alpha $)=0.05$, an intra-cluster correlation coefficient $($ ICC $)=0.02$, an effect size $=0.27$, the correlation coefficient between baseline and follow-up $(r)=0.3-0.5$, and power $=80-90 \%, 225-291$ participants would be needed at each of the intervention and control facilities in a CRCT. For this pilot study at three facilities, we intend to recruit 225-291 participants per facility. If we are able to recruit 20 perinatal women per week at each facility, we would need to spend at least 3 months doing recruitment.

\section{Patient file reviews}

During the baseline data collection period and throughout the implementation phase, fieldworkers will capture the outcome of the psychological distress questionnaire found in the MCRs of pregnant women attending healthcare facilities. The information obtained will be used to assess whether delivering the HSS interventions improved (i) detection and referral rates, (ii) uptake of the detection and referral interventions, and (iii) the sustainability of the detection and referral interventions.

\section{Documentation reviews}

Reviewing registers and referral forms will be used to assess coverage of the detection, referral and treatment interventions. Rates of detection and referral for women with CMDs and experiences of domestic violence will be assessed before the intervention, and monitored weekly throughout the intervention period, by triangulating information obtained from (i) a review of the daily patient registers completed by ANC nurses, and (ii) reviewing the referral forms completed by ANC nurses for patients who screen positive and agree to counselling. 
The proportion of perinatal women with CMDs and experiences of domestic violence who take up the referral and receive partial or full treatment will be assessed by triangulating information obtained from reviewing the referral and counselling feedback forms to be completed by OTLs for all patients who are referred for the task-sharing psychological counselling program.

Counselling competence

The counselling competence of the $\mathrm{CHWs}$ delivering the counselling intervention will be measured during their first three counselling sessions. OTLs will be tasked with completing a counselling session evaluation form while observing the counselling sessions. The evaluation form will use a Likert scale and consist of questions assessing their verbal and non-verbal skills, as well as a check list to evaluate the fidelity to the structured counselling format and content.

Qualitative assessments

Key informant interviews and focus group discussions with perinatal women, ANC nurses, CHWs and OTLs will be used to assess the acceptability, appropriateness, satisfaction and feasibility of the various HSS interventions.

\section{Ethical approval and consent to participate}

for the study has been obtained from the Human Research Ethics Committee at the University of Cape Town as well as from the Psychiatry, Nursing and Midwifery Research Ethics Subcommittee at King's College London. In addition, the Western Cape Department of Health approved the use of the research sites. All participants will be asked to provide written, informed consent after the procedure is verbally explained to them. Participants will be informed of their right to withdraw from the study at any time without consequences. Pregnant women who are recruited into the cohort study will receive a ZAR100 food voucher after completing all questionnaires at recruitment and again at the 36-week followup interview.

\section{Data handling and confidentiality}

All data will be processed in accordance with the General Data Protection Regulation 2018 (GDPR). Participants will be allocated a unique identifier, which will be used to maintain confidentiality and minimise the use of personal information. Questionnaires completed in hard copy will be stored in a locked room at the University of Cape Town. King's College London will be the data controller for the ASSET study.

\section{Data analyses}

A combination of mixed methods will be used to analyse the data collected. REDCap, a secure web platform for building and managing online databases and surveys, will be used to capture all quantitative data. Data will be exported to STATA/SE statistical software package version 15.1 (StataCorp., College Station, TX, USA) for analysis. Multiple imputation will be used to account for participants with incomplete data. Categorical variables will be described using frequency and percentages, and associations measured using Chi-square tests. Continuous variables will be described using means and standard deviations, and associations measured using t-tests. Linear regression models will be used to evaluate for change in EPDS scores, adjusting for the clinic and any potential confounders. Implementation outcomes will be evaluated using multivariable regression models that adjust for relevant confounders and clinic.

Qualitative data will be analysed using NVivo 12 Pro qualitative data analysis software (QSR International Pty Ltd) [42]. Semi-structured interviews will be transcribed by bilingual speakers. Transcripts will be analysed using a thematic analysis approach to generate initial codes and define, search for and review themes [43]. The development of initial 
codes will be guided to a certain degree by the interview topic guides. Further themes not captured by the initial coding will be identified through extensive reading of the transcripts and coding passages interpreted as important.

\section{Discussion}

There is a substantial treatment gap for perinatal women with depression, anxiety and exposure to domestic violence in low resource African settings. We selected HSS interventions to improve awareness, detection, referral and treatment of perinatal women experiencing symptoms of CMDs and domestic violence in Cape Town, South Africa. The purpose of this protocol is to describe how we will assess the effect of the HSS interventions on important implementation outcomes. The findings will help us refine the intervention components by identifying the barriers and facilitators experienced by the healthcare providers, their managers and the patients receiving the intervention.

The findings will also inform us about the feasibility and acceptability of a variety of implementation and clinical outcome measures in this context. Lessons learnt from the pilot study will be incorporated into the design of a cRCT for which further funding will be sought. The integration of detection, referral and care for perinatal women with depression, anxiety and exposure to domestic violence into routine low resource health facility and community settings is complex, and requires a careful evaluation of process and outcomes, if it is to be scaled up for broader population benefit.

\section{Abbreviations}

AFFIRM - Africa Focus on Intervention Research for Mental Health

ANC - antenatal care

BANC - basic antenatal care

CBS - community-based services

$\mathrm{CHW}$ - community health worker

CMD - common mental disorders

DoH - Department of Health

EPDS - Edinburgh postnatal depression scale

EPOC - Effective Practice and Organisation of Care

HSS - Health systems strengthening

KTU - knowledge translation unit

MOU - midwife obstetric unit

MCR - maternity case record

NPO - not-for-profit organisation

OTL - outreach team leader

PACK - practical approach to care kit 
ToC - theory of change

WC - Western Cape

\section{Declarations}

\section{Ethics approval and consent to participate}

Ethical approval for the study was obtained from the Human Research Ethics Committee at the University of Cape Town (Ref No: 139/2018) and the Psychiatry, Nursing and Midwifery Research Ethics Subcommittee at King's College London (Ref No: 17/18-7807). In addition, the Western Cape Department of Health approved the use of the research sites (Ref No: WC_201807_008 \& WC_201911_004). All participants provided written, informed consent after the procedure had been verbally explained to them and were informed that they were free to withdraw from the study at any time without consequences.

\section{Consent for publication}

Not applicable

\section{Availability of data and materials}

The datasets used and/or analysed during the current study are available from the corresponding author on reasonable request.

\section{Competing interests}

The authors declare that they have no competing interests

\section{Funding}

Funding was provided by the National Institute for Health Research (NIHR) Global Health Research Unit on Health System Strengthening in Sub-Saharan Africa, King's College London [GHRU 16/136/54]. The views expressed are those of the author(s) and not necessarily the NIHR. The NIHR did not play a role in the design of the study, collection or analysis of data, interpretation of findings or writing this manuscript.

\section{Authors' contributions}

All authors (ZA, SH, MS, PO, SB, NS, CL) contributed to the design of the study. ZA and CL conceptualised the idea for the paper. ZA drafted the paper and incorporated co-author comments and feedback. All authors reviewed the manuscript, provided input, and approved the final version.

\section{Acknowledgements}


We would like to thank the Western Cape Department of Health and the associated NPO's for their willingness to participate in this study and for their dedication to maternal mental health. We would like to thank the pregnant women for so generously giving their time.

\section{References}

1. City of Cape Town. Hanover Park - a public investment framework. 2015; Available at: http://resource.capetown.gov.za/documentcentre/Documents/City\%20strategies,\%20plans\%20and\%20frameworks /Hanover\%20Park\%20Public\%20Investment\%20Framework_22\%200ctober\%202015.pdf. Accessed December/05, 2017.

2. Abrahams Z, Lund C, Field S, Honikman S. Factors associated with household food insecurity and depression in pregnant South African women from a low socio-economic setting: a cross-sectional study. Soc Psychiatry Psychiatr Epidemiol 2018;53(4):363-372.

3. Lund C, Schneider M, Garman EC, Davies T, Munodawafa M, Honikman S, et al. Task-sharing of psychological treatment for antenatal depression in Khayelitsha, South Africa: Effects on antenatal and postnatal outcomes in an individual randomised controlled trial. Behav Res Ther 2020;130:103466.

4. Norman R, Bradshaw D, Schneider M, Jewkes R, Mathews S, Abrahams N, et al. Estimating the burden of disease attributable to interpersonal violence in South Africa in 2000. South African Medical Journal 2007;97(8):653-656.

5. Schneider M, Baron E, Davies T, Munodawafa M, Lund C. Patterns of intimate partner violence among perinatal women with depression symptoms in Khayelitsha, South Africa: A longitudinal analysis. Global Mental Health $2018 ; 5$.

6. Field S, Onah M, van Heyningen T, Honikman S. Domestic and intimate partner violence among pregnant women in a low resource setting in South Africa: a facility-based, mixed methods study. BMC Womens Health 2018;18(1):113.

7. van Heyningen T, Myer L, Onah M, Tomlinson M, Field S, Honikman S. Antenatal depression and adversity in urban South Africa. J Affect Disord 2016;203:121-129.

8. Ghimire U, Papabathini SS, Kawuki J, Obore N, Musa TH. Depression during Pregnancy and the Risk of Low Birth Weight, Preterm Birth and Intrauterine Growth Restriction-An updated Meta-analysis. Early Hum Dev 2020:105243.

9. Badr LK, Ayvazian N, Lameh S, Charafeddine L. Is the effect of postpartum depression on mother-infant bonding universal? Infant Behavior and Development 2018;51:15-23.

10. Department of Health. National Mental Health Policy Framework and Strategic Plan, 2013-2020 . 2013.

11. Department of Health. Maternity Case Record. 2018; Available at: https://pmhp.za.org/wpcontent/uploads/Western-Cape-Maternity-Case-Record-Version-19-June-2018.pdf. Accessed March/23, 2020.

12. Abrahams Z, Schneider M, Field S, Honikman S. Validation of a brief mental health screening tool for pregnant women in a low socio-economic setting. BMC psychology 2019;7(1):77.

13. Cornick R, Picken S, Wattrus C, Awotiwon A, Carkeek E, Hannington J, et al. The Practical Approach to Care Kit (PACK) guide: developing a clinical decision support tool to simplify, standardise and strengthen primary healthcare delivery. BMJ global health 2018;3(Suppl 5):e000962.

14. Abrahams Z, Boisits S, Schneider M, Honikman S, Lund C. Facilitators and barriers to detection and treatment of depression, anxiety and experiences of domestic violence in pregnant women attending Midwife Obstetric Units (MOUs) in Cape Town, South Africa: A qualitative study. 2021.

15. Honikman S, Van Heyningen T, Field S, Baron E, Tomlinson M. Stepped care for maternal mental health: a case study of the perinatal mental health project in South Africa. PLoS Med 2012;9(5):e1001222. 
16. Lund C, Schneider M, Davies T, Nyatsanza M, Honikman S, Bhana A, et al. Task sharing of a psychological intervention for maternal depression in Khayelitsha, South Africa: study protocol for a randomized controlled trial. Trials 2014;15(1):457.

17. Seward N, Murdoch J, Hanlon C, Araya R, Gao W, Harding R, et al. Implementation Science Protocol for a participatory, theory-informed implementation research programme in the context of health system strengthening in sub-Saharan Africa (ASSET-ImplementER). MedRxiv 2021.

18. Atkins L, Francis J, Islam R, O'Connor D, Patey A, Ivers N, et al. A guide to using the Theoretical Domains Framework of behaviour change to investigate implementation problems. Implementation Science 2017;12(1):1-18.

19. Pfadenhauer LM, Gerhardus A, Mozygemba K, Lysdahl KB, Booth A, Hofmann B, et al. Making sense of complexity in context and implementation: the Context and Implementation of Complex Interventions ( $\mathrm{CICl}$ ) framework. Implementation science 2017;12(1):1-17.

20. Effective Practice and Organisation of Care (EPOC). EPOC Taxonomy. 2015; Available at: https://epoc.cochrane.org/epoc-taxonomy. Accessed 23 September, 2021.

21. Proctor E, Silmere H, Raghavan R, Hovmand P, Aarons G, Bunger A, et al. Outcomes for implementation research: conceptual distinctions, measurement challenges, and research agenda. Administration and policy in mental health and mental health services research 2011;38(2):65-76.

22. Cotterill S, Knowles S, Martindale A, Elvey R, Howard S, Coupe N, et al. Getting messier with TIDieR: embracing context and complexity in intervention reporting. BMC medical research methodology 2018;18(1):1-10.

23. Seward N, Hanlon C, Abdulahi A, Abrams Z, Alem A, Araya R, et al. HeAlth System StrEngThening in four sub_Saharan African countries (ASSET) to achieve high-quality, evidence-informed surgical, maternal and newborn, and primary care: protocol for pre-implementation phase studies. 2021.

24. Hanlon C, Luitel NP, Kathree T, Murhar V, Shrivasta S, Medhin G, et al. Challenges and opportunities for implementing integrated mental health care: a district level situation analysis from five low-and middle-income countries. PloS one 2014;9(2):e88437.

25. Cox JL, Holden JM, Sagovsky R. Detection of postnatal depression: development of the 10-item Edinburgh Postnatal Depression Scale. Br J Psychiatry 1987;150(6):782-786.

26. Breuer $E$, Lee L, De Silva M, Lund C. Using theory of change to design and evaluate public health interventions: a systematic review. Implementation Science 2015;11(1):1-17.

27. HeAlth System StrEngThening in Sub-Saharan Africa (ASSET) Work Package 6 team. Maternal Mental Health Heath Promotion and Awareness. 2020; Available at: https://mfr.de-1.osf.io/render?url=https://osf.io/exh2t/? direct\%26mode=render\%26action=download\%26mode=render. Accessed 28 September, 2021.

28. Knowledge Translation Unit. PACK Overview. 2020; Available at: https://knowledgetranslation.co.za/pack/. Accessed 6 August, 2021.

29. HeAlth System StrEngThening in Sub-Saharan Africa (ASSET) Work Package 6 team. PACK Antenatal Women and Mental Health Module - Facility Trainers Manual. 2019; Available at: https://mfr.de-1.osf.io/render? url=https://osf.io/82wzc/?direct\%26mode=render\%26action=download\%26mode=render. Accessed 28 September, 2021.

30. Boisits S, Abrahams Z, Schneider M, Honikman S, Kaminer D, Lund C. Developing a task-sharing psychological intervention to treat mild to moderate symptoms of perinatal depression and anxiety in South Africa: a mixedmethod formative study. International Journal of Mental Health Systems 2021;15(1):1-14.

31. HeAlth System StrEngThening in Sub-Saharan Africa (ASSET) Work Package 6 team. Maternal Mental Health Counselling Skills for Community Health Workers. 2020; Available at: https://mfr.de-1.osf.io/render?

Page $17 / 20$ 
url=https://osf.io/jnfzd/?direct\%26mode=render\%26action=download\%26mode=render. Accessed 28 September, 2021.

32. Shea CM, Jacobs SR, Esserman DA, Bruce K, Weiner BJ. Organizational readiness for implementing change: a psychometric assessment of a new measure. Implementation science 2014;9(1):1-15.

33. Gabbidon J, Clement S, van Nieuwenhuizen A, Kassam A, Brohan E, Norman I, et al. Mental Illness: Clinicians' Attitudes (MICA) Scale-Psychometric properties of a version for healthcare students and professionals. Psychiatry Res 2013;206(1):81-87.

34. Stamm BH. The concise ProQOL manual. 2010.

35. Siegrist J, Starke D, Chandola T, Godin I, Marmot M, Niedhammer I, et al. The measurement of effort-reward imbalance at work: European comparisons. Soc Sci Med 2004;58(8):1483-1499.

36. Castell GS, Rodrigo CP, de la Cruz, Joy Ngo, Bartrina JA. Household food insecurity access scale (HFIAS). Nutricion hospitalaria 2015;31(3):272-278.

37. Ford-Gilboe M, Wathen CN, Varcoe C, MacMillan HL, Scott-Storey K, Mantler T, et al. Development of a brief measure of intimate partner violence experiences: the Composite Abuse Scale (Revised)-Short Form (CASR-SF). BMJ open 2016;6(12):e012824.

38. Üstün TB, Kostanjsek N, Chatterji S, Rehm J. Measuring health and disability: Manual for WHO disability assessment schedule WHODAS 2.0. : World Health Organization; 2010.

39. Zimet GD, Dahlem NW, Zimet SG, Farley GK. The multidimensional scale of perceived social support. J Pers Assess 1988;52(1):30-41.

40. Taylor A, Atkins R, Kumar R, Adams D, Glover V. A new Mother-to-Infant Bonding Scale: links with early maternal mood. Archives of women's mental health 2005;8(1):45-51.

41. Kohrt BA, Jordans MJ, Rai S, Shrestha P, Luitel NP, Ramaiya MK, et al. Therapist competence in global mental health: development of the ENhancing Assessment of Common Therapeutic factors (ENACT) rating scale. Behav Res Ther 2015;69:11-21.

42. Bazeley P, Jackson K. Qualitative data analysis with NVivo. 2013.

43. Gale NK, Heath G, Cameron E, Rashid S, Redwood S. Using the framework method for the analysis of qualitative data in multi-disciplinary health research. BMC medical research methodology 2013;13(1):1-8.

\section{Figures}




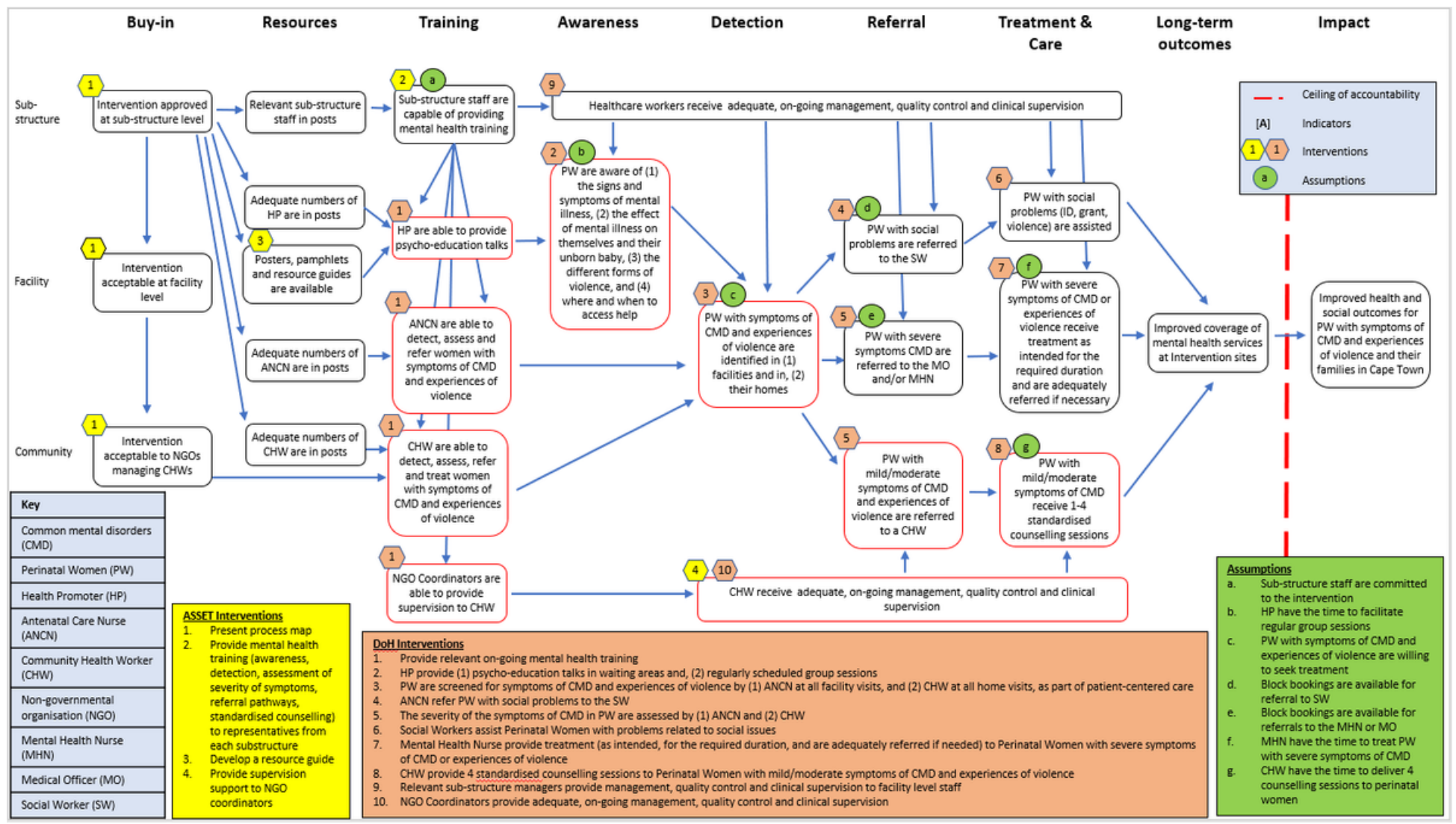

Figure 1

Theory of change diagram

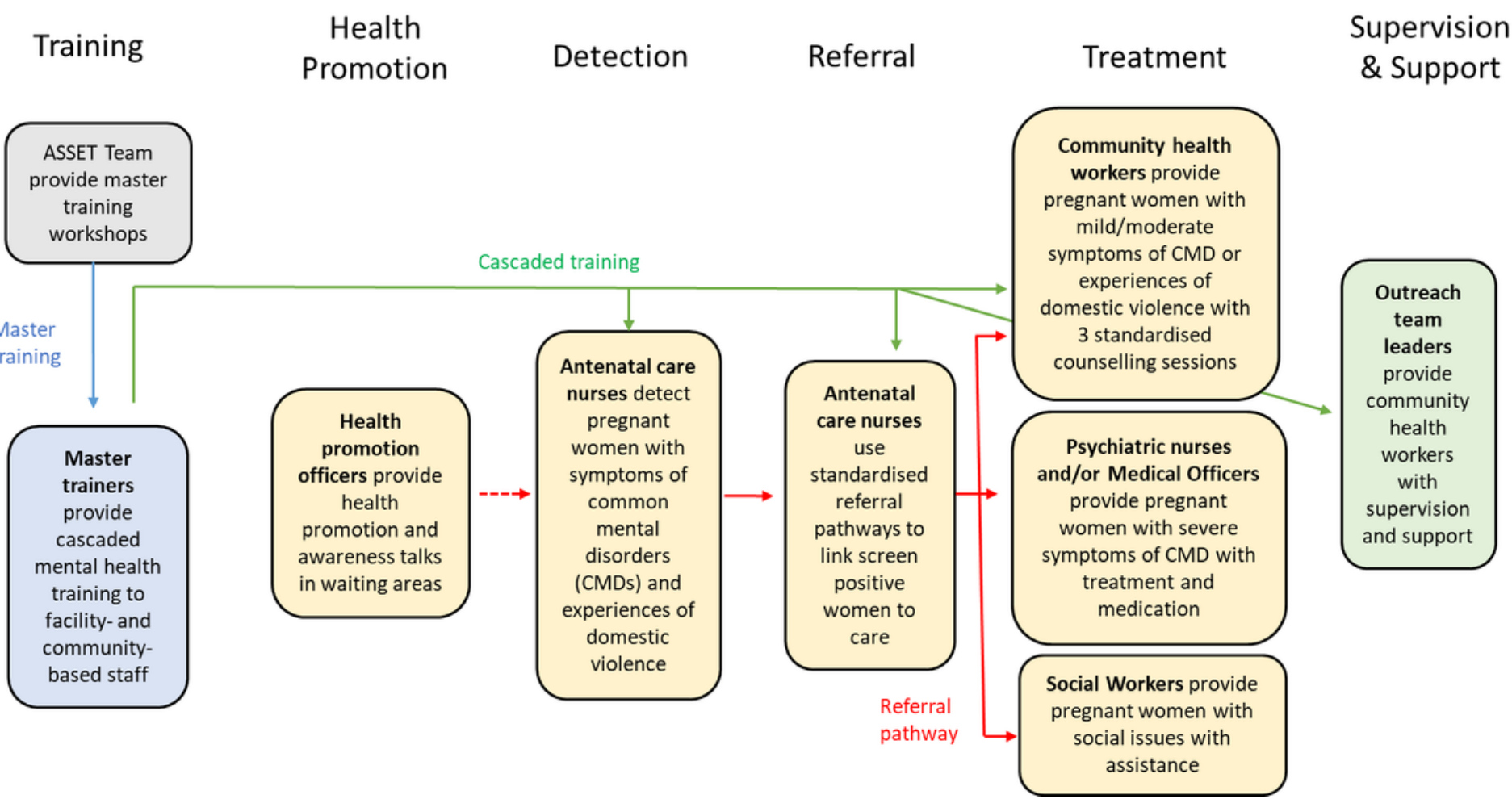

Figure 2 


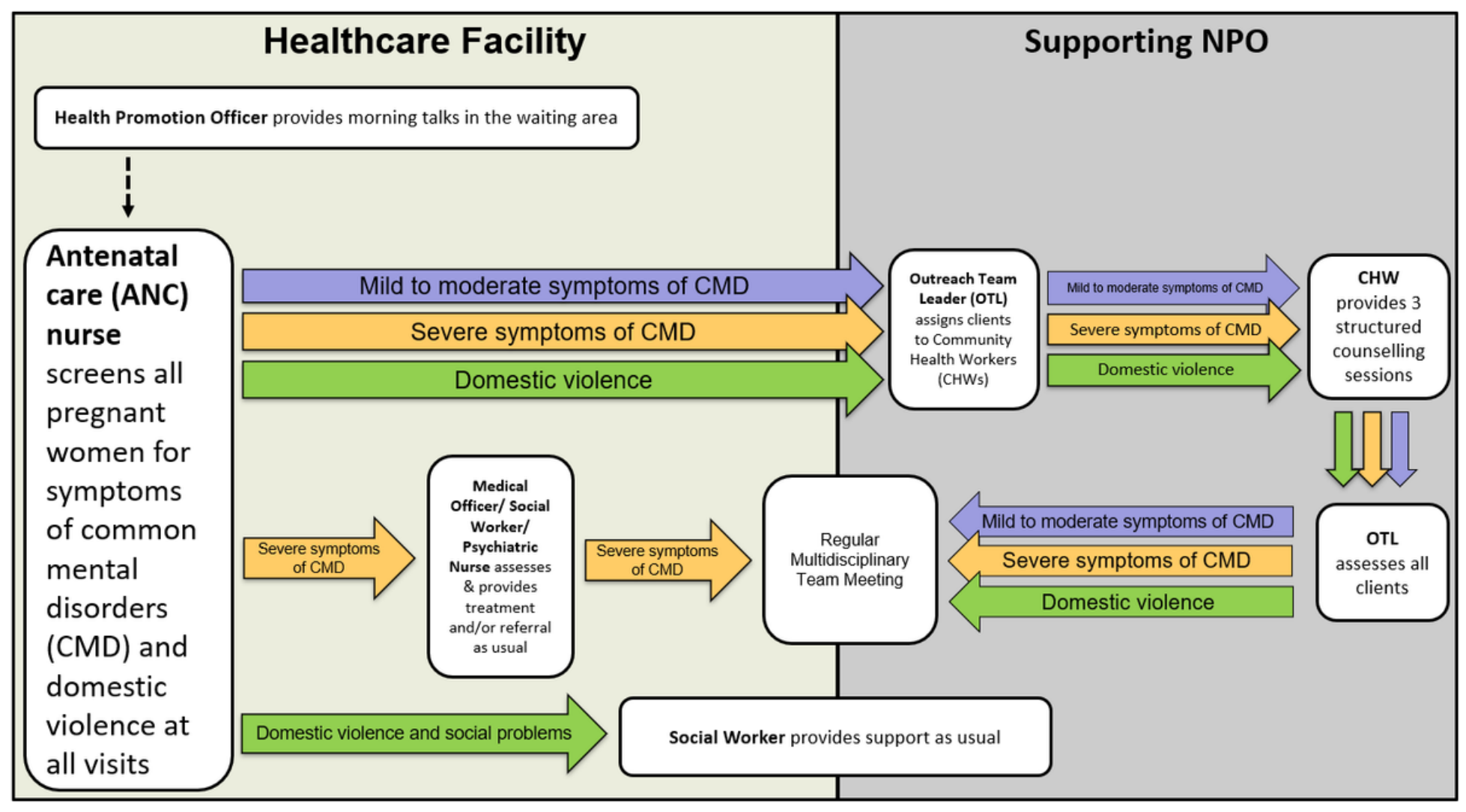

\section{Figure 3}

Referral pathway

\section{Supplementary Files}

This is a list of supplementary files associated with this preprint. Click to download.

- SupplementaryTable1.docx

- SupplementaryFigure2.docx 